\title{
The hyperbolic factor : a measure of decreasing impatience
}

Citation for published version (APA):

Rohde, K. I. M. (2005). The hyperbolic factor : a measure of decreasing impatience. METEOR, Maastricht University School of Business and Economics. METEOR Research Memorandum No. 047 https://doi.org/10.26481/umamet.2005047

Document status and date:

Published: 01/01/2005

DOI:

10.26481/umamet.2005047

Document Version:

Publisher's PDF, also known as Version of record

\section{Please check the document version of this publication:}

- A submitted manuscript is the version of the article upon submission and before peer-review. There can be important differences between the submitted version and the official published version of record.

People interested in the research are advised to contact the author for the final version of the publication, or visit the DOI to the publisher's website.

- The final author version and the galley proof are versions of the publication after peer review.

- The final published version features the final layout of the paper including the volume, issue and page numbers.

Link to publication

\footnotetext{
General rights rights.

- You may freely distribute the URL identifying the publication in the public portal. please follow below link for the End User Agreement:

www.umlib.nl/taverne-license

Take down policy

If you believe that this document breaches copyright please contact us at:

repository@maastrichtuniversity.nl

providing details and we will investigate your claim.
}

Copyright and moral rights for the publications made accessible in the public portal are retained by the authors and/or other copyright owners and it is a condition of accessing publications that users recognise and abide by the legal requirements associated with these

- Users may download and print one copy of any publication from the public portal for the purpose of private study or research.

- You may not further distribute the material or use it for any profit-making activity or commercial gain

If the publication is distributed under the terms of Article $25 \mathrm{fa}$ of the Dutch Copyright Act, indicated by the "Taverne" license above, 


\title{
The Hyperbolic Factor:
}

\section{a Measure of Decreasing Impatience ${ }^{1}$}

\author{
Kirsten I.M. Rohde ${ }^{2}$
}

November 1, 2005

${ }^{1}$ The author would like to thank Peter P. Wakker for many helpful discussions and comments. Han Bleichrodt also provided helpful comments.

${ }^{2}$ Department of Economics, Maastricht University, P.O. Box 616, 6200 MD Maastricht, The Netherlands. E-mail: K.Rohde@algec.unimaas.nl 


\begin{abstract}
Many studies have found that discounting is hyperbolic rather than constant. Hyperbolic discounting is becoming increasingly popular in economic applications. Most studies that provide evidence in favor of hyperbolic discounting either are merely qualitative or they depend on assumptions about, or parametric fittings of, utility functions. This paper provides a quantitative measure for the degree of deviation from stationarity that can overcome the problems mentioned. This measure, the hyperbolic factor, can easily be calculated from data and does not require knowledge of the utility function. Moreover, it provides simple preference foundations of the most popular discount functions. Thus, the hyperbolic factor provides an easy tool for theoretical preference foundations, critical empirical tests, and quantitative measurements of hyperbolic discounting.
\end{abstract}

Keywords: Hyperbolic discounting, hyperbolic factor, time preference

JEL classification: D90 


\section{Introduction}

Since Samuelson's (1937) introduction of constant discounted utility, this model has been widely accepted as a normative and descriptive model of intertemporal choice. According to general, possibly non-constant, discounted utility, a stream of outcomes is evaluated by first determining the utility of every outcome, i.e. the value of the outcome would it have been received immediately, and then multiplying each utility by a discount factor that corresponds to the time-point of receipt. Constant discounting implies that a preference between two streams of outcomes is not affected if all outcomes in both streams are delayed by the same time interval.

Recently, there has been an increasing number of empirical studies suggesting that discounting is not constant, including Benzion, Rapoport, and Yagil (1989), Bleichrodt and Johannesson (2001), Cairns and van der Pol (2000), Green, Fristoe, and Myerson (1994), Kirby and Marakovic (1995), Mazur (1987, 2001), Read and Read (2004), Rodriguez and Logue (1988), and Thaler (1981). If an early reward and another, later and larger reward are perceived as being equivalent, then delaying both rewards equally will, for most people and animals, result in a strict preference for the later and larger reward, revealing decreasing impatience.

As a consequence of decreasing impatience, individuals' preferences can be dynamically inconsistent. Consider a person who prefers to receive two apples in one year plus one day rather than one apple in one year, but prefers to receive one apple today rather than two apples tomorrow (Thaler, 1981). This person is decreasingly impatient. If his preferences between 'today' and 'tomorrow' remain the same for one year, then in one year from 
now, he will prefer to receive one apple on that day rather than two apples one day later. Thus, his preferences between the two options will have changed over time. In this sense decreasing impatience may be viewed as reflecting an irrationality.

An increasingly popular model that captures decreasing impatience is hyperbolic discounting (Loewenstein and Prelec, 1992; Harvey, 1986; Mazur, 1987). This model has been used in many fields (Akerlof, 2002; Harris and Laibson, 2001; Krusell and Smith, 2003; Laibson, 1997; Luttmer and Mariotti, 2003; O'Donoghue and Rabin, 1999; Thaler and Benartzi, 2004). Most studies that provide empirical evidence in favor of hyperbolic discounting assume a particular, often linear, utility function or first need to parametrically fit utility. Thus, the quantitative evidence in favor of hyperbolic discounting is confounded by assumptions about and parametric fittings of utility. Most qualitative studies in favor of hyperbolic discounting only reject constant discounting and provide evidence in favor of general decreasing impatience, not of hyperbolic discounting in particular.

This paper proposes a simple method to quantify the degree of deviation from stationarity that does not need assumptions about or estimations of utility. That is, a measure of decreasing impatience is introduced, the hyperbolic factor, which can easily be calculated from data without knowledge of utility.

One approach to construct a measure of decreasing impatience would be to find out how impatience changes over time. This would require knowledge of impatience at each timepoint, i.e. knowledge of the discount function. Then, to determine this discount function, we would also need to know the utility function. It is, indeed, commonly believed in the field that such a procedure should be followed. Surprisingly, as this paper shows, we do not need to go through all these steps. In fact, measuring the degree of decreasing impatience 
is even easier than measuring the discount function.

Our method of measurement is similar to the utility measurement method of Wakker and Deneffe (1996). There, choices between gambles under uncertainty are constructed in such a manner that subjective probabilities or decision weights cancel from the equations, so that utilities can be measured without the need to measure subjective or weighted probabilities. Similarly, this paper constructs choices between delayed outcomes in such a manner that the absolute level of discounting and the utilities of outcomes cancel from the equations, so that we can measure variations in impatience and, thus, degrees of irrationality, without knowledge of utility or the absolute level of discounting.

As will be shown, the hyperbolic factor is a useful tool in the axiomatization of the various discount functions that exist today. A constant positive hyperbolic factor corresponds to generalized hyperbolic discounting (Loewenstein and Prelec, 1992). Quasi-hyperbolic discounting holds if and only if the hyperbolic factor is equal to zero for all points in time except the present (Phelps and Pollak, 1968). If, in addition, the hyperbolic factor is zero in the present, then constant discounting holds. Thus, estimating the hyperbolic factor and testing whether it is constant will be useful in testing which of the currently used models fit empirical data best and in testing whether these existing models are appropriate at all or whether different models need to be developed.

Prelec (2004) introduced another measure of decreasing impatience. Relative to the hyperbolic factor, his measure is more complicated: it uses the second derivative of the logarithm of the discount function, which can only be obtained after complex measurements of discounting and utility. Moreover, his measure is not constant under hyperbolic discounting. The difference between Prelec's measure and the hyperbolic factor is analogous to the 
difference between the measure of absolute and that of relative risk aversion (Mas-Colell, Whinston, and Green, 1995). While the former is most useful for CARA-utility (constant absolute risk aversion), the latter is most useful for CRRA-utility (constant relative risk aversion). Similarly, while the hyperbolic factor is most useful for hyperbolic discounting, Prelec's measure will be more useful for other discount functions. Finally, the hyperbolic factor is model-free, i.e. it can also be used as a measure of decreasing impatience when preferences cannot be represented by discounted utility. This is not the case for Prelec's measure, which essentially needs a discount function.

Section 2 defines the hyperbolic factor. This factor is applied to discounted utility in Section 3. All proofs are in the Appendix.

\section{The Hyperbolic Factor Defined}

Let $\mathcal{X}=\mathbb{R}^{m}$ be a set of outcomes ${ }^{1}$ and $\mathcal{T}=\mathbb{R}_{+}$a set of time-points. A timed outcome $(t, \mu)$ yields outcome $\mu$ at time $t$ and nothing $(=0)$ at all other points in time, where $t=0$ corresponds to 'today'. We examine preferences $\succcurlyeq$ over timed outcomes. The relations $\preccurlyeq, \succ, \prec, \sim$ are as usual. Preferences over outcomes are derived from preferences over timed outcomes consumed today, i.e. $\chi \succcurlyeq \mu$ if and only if $(0, \chi) \succcurlyeq(0, \mu)$.

We assume that $\succcurlyeq$ is a weak order, i.e $\succcurlyeq$ is complete $((s, \mu) \succcurlyeq(t, \chi)$ or $(t, \chi) \succcurlyeq(s, \mu)$ for all $\mu, \chi \in \mathcal{X}$ and $s, t \in \mathcal{T}$, possibly both) and transitive. Preferences are monotonic if $\chi \succcurlyeq \mu$ implies $(t, \chi) \succcurlyeq(t, \mu)$ for every $t \in \mathcal{T}$, and $\chi \succ \mu \operatorname{implies}(t, \chi) \succ(t, \mu)$

\footnotetext{
${ }^{1}$ All results in this paper remain valid if $\mathcal{X}$ is a connected topological space containing a reference outcome 'nothing.' $\mathcal{X}$ can, for instance, be any convex subset of $\mathbb{R}^{m}$ containing zero, or a set of non-quantified health states.
} 
for every $t \in \mathcal{T}$. Preferences are impatient if for every $s<t, \chi \succ 0 \operatorname{implies}(s, \chi) \succ$ $(t, \chi)$ and $\chi \prec 0$ implies $(s, \chi) \prec(t, \chi)$. Preferences are continuous if for every $(t, \chi)$ the sets $\{(s, \mu) \in \mathcal{T} \times \mathcal{X} \mid(s, \mu) \succcurlyeq(t, \chi)\}$ and $\{(s, \mu) \in \mathcal{T} \times \mathcal{X} \mid(s, \mu) \preccurlyeq(t, \chi)\}$ are closed. Throughout this paper we assume that preferences constitute a continuous, monotonic, and impatient weak order.

Consider two equivalent timed outcomes $(s, \mu) \sim(t, \chi)$, with $s<t$. Then we have either $\chi \succ \mu \succ 0$ or $\chi \prec \mu \prec 0$ ( $\mu$ is 'moderate' and $\chi$ is 'extreme'). If the outcome $\mu$ is delayed by an interval $\tau$, then stationarity implies that the outcome $\chi$ should also be delayed by $\tau$ in order to maintain indifference. Thus, under stationarity $(s, \mu) \sim(t, \chi)$ implies $(s+\tau, \mu) \sim(t+\tau, \chi)$. Stationarity reflects constant impatience.

The preference relation $\succcurlyeq$ exhibits decreasing impatience if for all $s<t, \tau \in \mathcal{T}$, (i) $\chi \succ \mu \succ 0$ and $(s, \mu) \sim(t, \chi)$ imply $(t+\tau, \chi) \succcurlyeq(s+\tau, \mu)$, and (ii) $\chi \prec \mu \prec 0$ and $(s, \mu) \sim(t, \chi)$ imply $(t+\tau, \chi) \preccurlyeq(s+\tau, \mu)$; increasing impatience holds if the implied preferences are always the reverse. Thus, with decreasing impatience, when we consider two equivalent timed outcomes, then delaying both outcomes equally will result in less distinction between the time-points, and, thus, more preference for the timed outcome with the preferred outcome. In this sense, decreasing impatience reflects that a time difference becomes decreasingly important as it lies farther in the future. Assume another preference relation $\succcurlyeq^{*}$, which also is a continuous, monotonic and impatient weak order. Preferences $\succcurlyeq^{*}$ exhibit more decreasing impatience than $\succcurlyeq$ if for all $s<t, \tau, \sigma \in \mathcal{T}$ and $\mu \nsim \chi$, (i) $\chi^{*} \succ^{*} \mu^{*} \succ^{*} 0,(s, \mu) \sim(t, \chi),(s+\sigma, \mu) \sim(t+\tau, \chi)$, and $\left(s, \mu^{*}\right) \sim^{*}\left(t, \chi^{*}\right)$ imply $\left(t+\tau, \chi^{*}\right) \succcurlyeq^{*}\left(s+\sigma, \mu^{*}\right)$, and (ii) $\chi^{*} \prec^{*} \mu^{*} \prec^{*} 0,(s, \mu) \sim(t, \chi),(s+\sigma, \mu) \sim(t+\tau, \chi)$, and $\left(s, \mu^{*}\right) \sim^{*}\left(t, \chi^{*}\right)$ imply $\left(t+\tau, \chi^{*}\right) \preccurlyeq^{*}\left(s+\sigma, \mu^{*}\right)$ (Prelec, 2004). 
Consider again two equivalent timed outcomes $(s, \mu) \sim(t, \chi)$ with $s<t$. Assume that $(s+\sigma, \mu) \sim(t+\tau, \chi)$. Decreasing (increasing) impatience implies that $\tau-\sigma>0(\tau-\sigma<0)$. An obvious measure of decreasing impatience is, therefore, $\tau-\sigma$. This measure $\tau-\sigma$, however, will depend on $s, t, \sigma, \mu$, and $\chi$, and will be hard to compare across different outcomes and time-points. The main purpose of this paper is to propose a transformation of this measure that is better suited as a measure of impatience, and that can be compared more easily across different outcomes and time-points. This proposed measure, the hyperbolic factor, is defined next. It is just as easily observable from preferences as $\tau-\sigma$ itself. Unlike $\tau-\sigma$, however, it will be constant, i.e. independent of $s, t, \sigma, \mu$ and $\chi$, for all hyperbolic discounting models currently used in the literature, as we will see in Section 3. Outcomes $\mu, \chi \in \mathcal{X}$ and time-points $s, t, \sigma, \tau \in \mathcal{T}$, with $s<t, \tau>0$, form an indifference pair if

$$
(s, \mu) \sim(t, \chi) \text { and }(s+\sigma, \mu) \sim(t+\tau, \chi) .
$$

\section{Definition 2.1}

For every indifference pair as in eq. 1 the hyperbolic factor is defined as

$$
\frac{\tau-\sigma}{t \sigma-s \tau}
$$

For general preferences, a hyperbolic factor may not always be defined for every outcome $\chi$ and all $s<t$ and $\tau>0$. For instance, there may be no $\mu$ and $\sigma$ that satisfy eq. 1 . Our assumptions about preferences imply that such a case can never arise, so that a $\mu$ and $\sigma$ as described can always be found. This claim is formalized in the next theorem.

Theorem 2.2 For every $\chi \nsim 0, s<t$, and $\tau>0$, there are $a \mu$, and a unique $\sigma$ such that an indifference pair as in eq. 1 results. 
Now we can define the function $H$ for every $\chi \nsim 0, s<t$, and $\tau>0$, as

$$
H(s, t, \chi, \tau)=\frac{\tau-\sigma}{t \sigma-s \tau}
$$

where $\sigma$ is such that together with a $\mu$ and the arguments of $\mathrm{H}$, it yields an indifference pair as in eq. 1. The function $H$ gives the hyperbolic factors. In general, $H$ need not always be regular, i.e. $H$ is infinite if $t \sigma=s \tau$, and negative in spite of strongly decreasing impatience if $t \sigma<s \tau$. Yet, as we will see later, for all discounted utility models popular in the literature, regularity holds, i.e. for every indifference pair as in eq. 1 we have $t \sigma>s \tau$.

Note that for every indifference pair, a hyperbolic factor can be calculated. From $n$ indifferences as in eq. 1 with varying time-points, $\left(\begin{array}{l}n \\ 2\end{array}\right) / 2$ hyperbolic factors can be calculated. Non-negative hyperbolic factors correspond to decreasing impatience. We will see in Section 3 that hyperbolic discounting induces non-negative hyperbolic factors, and, thus, decreasing impatience.

Theorem 2.3 Let regularity hold. Preferences $\succcurlyeq$ exhibit decreasing impatience if and only if $H \geq 0$. Preferences $\succcurlyeq$ exhibit increasing impatience if and only if $H \leq 0$.

The hyperbolic factor also serves as a measure of decreasing impatience, as shown in the next theorem. Thus, it properly captures Prelec's (2004) relative decreasing impatience. When we consider another preference relation $\succcurlyeq^{*}$, then it is assumed that $\succcurlyeq^{*}$ is a continuous, monotonic and impatient weak order, and that the corresponding hyperbolic factors are given by $H^{*}(s, t, \chi, \tau)$.

Theorem 2.4 Let regularity hold. Preferences $\succcurlyeq^{*}$ exhibit more decreasing impatience than $\succcurlyeq$ if and only if $H^{*}\left(s, t, \chi^{*}, \tau\right) \geq H(s, t, \chi, \tau)$ for all $s, t, \tau, \chi, \chi^{*}$. 
Thus, we have shown that the hyperbolic factor is an appropriate model-free measure of decreasing impatience that can easily be obtained from an indifference pair.

\section{The Hyperbolic Factor and Discounted Utility}

Discounted utility holds if there exist a discount function $\phi$ and a utility function $u$ such that preferences $\succcurlyeq$ can be represented by

$$
D U(t, \mu)=\phi(t) u(\mu)
$$

where $\phi$ is continuous and strictly decreasing, $\phi(0)=1, \phi(t)>0$ for every $t$, and $u$ is continuous, $u(0)=0$, and there is an outcome $\chi \in \mathcal{X}$ with $u(\chi) \neq 0$. Fishburn and Rubinstein (1982) characterized discounted utility. In this section we will assume that discounted utility holds. Thus, preferences still constitute a continuous, monotonic and impatient weak order as in Section 2. We will not assume regularity, but instead derive it later from other assumptions.

Under discounted utility, the hyperbolic factor is independent of the outcomes, as the following theorem shows.

Theorem 3.1 Let discounted utility hold. Then $H(s, t, \chi, \tau)$ is independent of $\chi$.

Two decision-makers with different discount functions $\phi$ and $\phi^{*}$ that are related by a power transformation $\phi^{*}(t)=[\phi(t)]^{c}$ have equal hyperbolic factors, as stated in Observation 3.2. Thus, in order to measure deviations from stationarity, we do not even need to know how much people discount in an absolute sense. This observation underlies the possibility to analyze decreasing impatience without a need to measure the discount function or utility. 
Observation 3.2 Let discounted utility hold. Consider two discount functions $\phi$ and $\phi^{*}$ with corresponding $H$ and $H^{*}$. If there is a $c \in \mathbb{R}$ such that $\phi^{*}(t)=[\phi(t)]^{c}$ then $H^{*}\left(s, t, \chi^{*}, \tau\right)=H(s, t, \chi, \tau)$ for every $s, t, \tau, \chi, \chi^{*}$.

\subsection{Constant Discounting}

For a long time, constant discounting has been a traditional assumption in economics. Preferences satisfy constant discounting if there is a constant discount factor $\delta$ such that $\phi(t)=\delta^{t}$ for every $t$. Constant discounting is equivalent to the hyperbolic factor always being zero.

Theorem 3.3 The following two statements are equivalent under discounted utility.

(i) Preferences $\succcurlyeq$ satisfy constant discounting.

(ii) $H(s, t, \chi, \tau)=0$ for all $s, t, \chi, \tau$.

\subsection{Generalized Hyperbolic Discounting}

Following up on the empirical studies that found violations of stationarity, Loewenstein and Prelec (1992) introduced the generalized hyperbolic discount function, which is defined by

$$
\phi(t)=(1+h t)^{-r / h}
$$

with $h>0, r>0$. Generalized hyperbolic discounting is equivalent to the hyperbolic factor being a positive constant.

Theorem 3.4 The following two statements are equivalent under discounted utility. 
(i) Preferences $\succcurlyeq$ satisfy generalized hyperbolic discounting $\phi(t)=(1+h t)^{-r / h}$ with parameter $h>0$.

(ii) There is a constant $h>0$ such that $H(s, t, \chi, \tau)=h$ for all $s, t, \chi, \tau$.

Mazur (1987) tested a necessary condition for hyperbolic discounting that also did not require knowledge of utility. We provide a testable condition that is not only necessary, but also sufficient for hyperbolic discounting, as Theorem 3.4 shows.

\subsection{Harvey Discounting}

Harvey (1986) proposed a discount function given by

$$
\phi(t)=(1+t)^{-r}
$$

This Harvey discounting is equivalent to generalized hyperbolic discounting with a hyperbolic factor that equals one.

Theorem 3.5 The following two statements are equivalent under discounted utility.

(i) Preferences $\succcurlyeq$ satisfy Harvey discounting.

(ii) $H(s, t, \chi, \tau)=1$ for all $s, t, \chi, \tau$.

\subsection{Mazur Discounting}

Mazur (1987) proposed a discount function given by

$$
\phi(t)=(1+h t)^{-1}
$$

This Mazur discounting is equivalent to generalized hyperbolic discounting with $r=h$. 
It follows that the hyperbolic factor does not distinguish between the generalized hyperbolic discounting of Loewenstein and Prelec (1992) and the model of Mazur (1987). This is because the hyperbolic factor only restricts the parameter $h$ and not the parameter $r$ as we saw in Observation 3.2. Indeed, on our domain of timed outcomes the two models cannot be distinguished, because they differ only regarding the absolute level of discounting and not regarding the degree of decreasing impatience. This finding reflects once more that changes in impatience and the corresponding irrationalities can be investigated independently of the absolute level of discounting.

\subsection{Quasi-hyperbolic Discounting}

Phelps and Pollak (1968) introduced quasi-hyperbolic discounting, as used by Laibson (1997) and many others. The quasi-hyperbolic discount function is given by

$$
\phi(t)= \begin{cases}1 & \text { if } t=0 \\ \beta \delta^{t} & \text { if } t>0 .\end{cases}
$$

for some $\beta \leq 1$, and some $\delta>0$.

Quasi-hyperbolic discounting is equivalent to stationarity for all of the future except the present.

Theorem 3.6 The following two statements are equivalent under discounted utility.

(i) Preferences $\succcurlyeq$ satisfy quasi-hyperbolic discounting.

(ii) $H(s, t, \chi, \tau)=0$ for all $s>0, t, \chi, \tau$.

Thus, when combined with Fishburn and Rubinstein's (1982) preference foundation of discounted utility, this section provided preference foundations for all currently popular 
discount models.

\section{Conclusion}

This paper has introduced the hyperbolic factor, a quantitative measure of decreasing impatience, which can easily be obtained from an indifference pair. In addition to being a simple measure, the hyperbolic factor is useful in characterizing all popular discount models. Hyperbolic discounting holds if and only if the hyperbolic factor is constant and positive. The discount function of Harvey applies if and only if the hyperbolic factor is always equal to one. Quasi-hyperbolic discounting holds if and only if the hyperbolic factor is equal to zero for all future points in time except the present. If, in addition, the hyperbolic factor is equal to zero today, then constant discounting holds.

A direction for future research is to calculate hyperbolic factors from data, which will illustrate how strong the evidence in favor of hyperbolic discounting is. A major advantage of such future studies, in comparison to earlier ones, is that they will not be confounded by assumptions about or estimations of instant utility functions. Testing whether hyperbolic factors are constant and positive will indicate whether hyperbolic discounting is the appropriate alternative to constant discounting.

\section{Appendix}

\section{Proof of Theorem 2.2}

Consider $s, t, \chi, \tau$ with $0 \leq s<t, \chi \nsim 0$, and $\tau>0$. Assume that $\chi \succ 0$. By monotonicity 
and impatience we know that ${ }^{2}$

$$
(s, 0) \sim(t, 0) \prec(t, \chi) \prec(s, \chi) .
$$

By continuity and connectedness of $\mathbb{R}^{m}$ there must then be a $\mu$ with $(s, \mu) \sim(t, \chi)$ and $\chi \succ \mu \succ 0$. Thus,

$$
(s, \mu) \sim(t, \chi) \succ(t+\tau, \chi) \succ(t+\tau, \mu) .
$$

By continuity there must then be a $\sigma$ with $(s+\sigma, \mu) \sim(t+\tau, \chi)$. By replacing all ' $\succ$ ' by ' $\prec$ ' and all ' $\prec$ ' by ' $\succ$ ', this reasoning shows that similar things hold for $\chi \prec 0$.

By monotonicity and impatience, $\mu$ is unique up to indifference and $\sigma$ is unique.

\section{Proof of Theorem 2.3}

By regularity, we have $H \geq 0$ if and only if $\tau-\sigma \geq 0$ for all indifference pairs as in eq. 1 . Thus, by Theorem 2.2, we have $H \geq 0$ if and only if decreasing impatience holds.

\section{Proof of Theorem 2.4}

By regularity, we have $H^{*}\left(s, t, \chi^{*}, \tau\right) \geq H(s, t, \chi, \tau)$ for every $s, t, \tau, \chi, \chi^{*}$ if and only if $\sigma^{*} \leq \sigma$ for all $s<t, \chi \nsim 0, \tau>0$ with $(s, \mu) \sim(t, \chi),(s+\sigma, \mu) \sim(t+\tau, \chi)$ and $\left(s, \mu^{*}\right) \sim^{*}\left(t, \chi^{*}\right),\left(s+\sigma^{*}, \mu^{*}\right) \sim^{*}\left(t+\tau, \chi^{*}\right)$, which, by impatience, holds if and only if $\succcurlyeq^{*}$ exhibits more decreasing impatience than $\succcurlyeq$.

\footnotetext{
${ }^{2}$ By the definition of a timed outcome it follows that $(s, 0) \sim(t, 0)$ for every $s, t$.
} 


\section{Proof of Theorem 3.1}

Let $H(s, t, \chi, \tau)=h$ and $H\left(s, t, \chi^{*}, \tau\right)=h^{*}$. Then there are $\mu, \sigma, \mu^{*}, \sigma^{*}$, with $(s, \mu) \sim(t, \chi)$, $(s+\sigma, \mu) \sim(t+\tau, \chi),\left(s, \mu^{*}\right) \sim\left(t, \chi^{*}\right)$ and $\left(s+\sigma^{*}, \mu^{*}\right) \sim\left(t+\tau, \chi^{*}\right)$. By discounted utility it follows that

$$
\phi(s) u(\mu)=\phi(t) u(\chi)
$$

and

$$
\phi(s+\sigma) u(\mu)=\phi(t+\tau) u(\chi) .
$$

Therefore,

$$
\frac{u(\mu)}{u(\chi)}=\frac{\phi(t)}{\phi(s)}=\frac{\phi(t+\tau)}{\phi(s+\sigma)}
$$

Similarly,

$$
\frac{u\left(\mu^{*}\right)}{u\left(\chi^{*}\right)}=\frac{\phi(t)}{\phi(s)}=\frac{\phi(t+\tau)}{\phi\left(s+\sigma^{*}\right)}
$$

By impatience it then follows that $\sigma^{*}=\sigma$ and $h=h^{*}$. This proves our result.

A similar reasoning proves Observation 3.2.

\section{Proof of Theorem 3.3}

Let $H(s, t, \chi, \tau)=0$ for all $s, t, \chi, \tau$. Then

$$
(s, \mu) \sim(t, \chi)
$$

if and only if

$$
(s+\tau, \mu) \sim(t+\tau, \chi),
$$


i.e. stationarity holds. Thus, for every $s, t, \sigma \in \mathcal{T}$,

$$
\frac{\phi(s)}{\phi(t)}=\frac{\phi(s+\tau)}{\phi(t+\tau)}
$$

Therefore, by setting $s=0$, for every $t, \tau \in \mathcal{T}$,

$$
\phi(t) \phi(\tau)=\phi(t+\tau)
$$

By Cauchy's functional equation it follows that there must be a $c \in \mathbb{R}$ such that $\phi(t)=e^{c t}$ for every $t \in \mathcal{T}$. Now let $\delta=e^{c}$. Then, $\phi(t)=\delta^{t}$. The converse follows easily.

\section{Proof of Theorem 3.4}

Let there be a constant $h>0$ such that $H(s, t, \chi, \tau)=h$ for all $s, t, \chi, \tau$. Assume that

$$
u(\mu)=\phi(t) u(\chi) \text { and } \phi(\sigma) u(\mu)=\phi(t+\tau) u(\chi),
$$

with $t>0$. Then we must have

$$
\frac{\tau-\sigma}{t \sigma}=h
$$

Let $k=1+h t$. It follows that $t+\tau=t+k \sigma$. Moreover, $k$ is a constant that depends only on $t$. Loewenstein and Prelec (1992) showed that this implies that the discount function is of the generalized hyperbolic form. Thus, there must be parameters $\tilde{h}, r$ such that $\phi(t)=(1+\tilde{h} t)^{-r / \tilde{h}}$. It follows that $\tilde{h}=h$. From the assumption that $\phi(\cdot)$ is strictly decreasing it follows that $r>0$. This proves one direction of our result. The proof of the other direction is straightforward. 


\section{Proof of Theorem 3.5}

Harvey discounting is equivalent to generalized hyperbolic discounting with $h=1$.

\section{Proof of Theorem 3.6}

Let $H(s, t, \chi, \tau)=0$ for all $s>0, t, \chi, \tau$. Then for every $s, t, \sigma \in \mathcal{T}$, with $s, t>0$

$$
\frac{\phi(s)}{\phi(t)}=\frac{\phi(s+\sigma)}{\phi(t+\sigma)}
$$

Let $s_{0}>0$. Define the function $\phi_{0}$ on $\mathcal{T}$ by $\phi_{0}(t)=\phi\left(t+s_{0}\right) / \phi\left(s_{0}\right)$. Then for every $s, t, \sigma \in \mathcal{T}$

$$
\frac{\phi_{0}(s)}{\phi_{0}(t)}=\frac{\phi\left(s+s_{0}\right)}{\phi\left(t+s_{0}\right)}=\frac{\phi\left(s+s_{0}+\sigma\right)}{\phi\left(t+s_{0}+\sigma\right)}=\frac{\phi_{0}(s+\sigma)}{\phi_{0}(t+\sigma)} .
$$

Moreover, $\phi_{0}(0)=1$. By Cauchy's functional equation it follows that there must be a $c_{0} \in \mathbb{R}$ such that $\phi_{0}(t)=e^{c_{0} t}$ for every $t \in \mathcal{T}$. Therefore, $\phi\left(t+s_{0}\right)=e^{c_{0} t} \phi\left(s_{0}\right)$ for every $t \in \mathcal{T}$. Thus, $\phi(t)=e^{c_{0}\left(t-s_{0}\right)} \phi\left(s_{0}\right)$ for all $t \geq s_{0}$. Define $\delta_{0}=e^{c_{0}}$ and $\beta_{0}=e^{-c_{0} s_{0}} \phi\left(s_{0}\right)$. Then $\phi(t)=\beta_{0} \delta_{0}^{t}$ for all $t \geq s_{0}$. Similarly, consider an $s_{1}$ with $0<s_{1}<s_{0}$ and with corresponding $\beta_{1}$ and $\phi_{1}$. It follows that for all $t \geq s_{0}, \phi(t)=\beta_{1} \delta_{1}^{t}=\beta_{0} \delta_{0}^{t}$, so $\beta_{0}=\beta_{1}$ and $\delta_{0}=\delta_{1}$. We can continue this argument repeatedly.

Thus, letting $\beta=\beta_{0}$ and $\delta=\delta_{0}$ we obtain $\phi(t)=\beta \delta^{t}$ for all $t>0$. By definition we have $\phi(0)=1$.

\section{References}

Akerlof, George A. (2002), "Behavioral Macroeconomics and Macroeconomic Behavior," American Economic Review, 92, 411-433. 
Benzion, Uri, Amnon Rapoport, and Joseph Yagil (1989), "Discount Rates Inferred from Decisions: An Experimental Study," Management Science, 35, 270-284.

Bleichrodt, Han, and Magnus Johannesson (2001), "Time Preference for Health: A Test of Stationarity versus Decreasing Timing Aversion," Journal of Mathematical Psychology, 45, 265-282.

Cairns, John and Marjon van der Pol (2000), "Valuing Future Private and Social Benefits: The Discounted Utility Model versus Hyperbolic Discounting Models," Journal of Economic Psychology, 21, 191-205.

Fishburn, Peter C., and Ariel Rubinstein (1982), "Time Preference," International Economic Review, 23, 677-694.

Green, Leonard, Nathanael Fristoe, and Joel Myerson (1994), "Temporal Discounting and Preference Reversals in Choice between Delayed Outcomes," Psychonomic Bulletin and Review, 1, 383-389.

Harris, Christopher, and David Laibson (2001), "Dynamic Choices of Hyperbolic Consumers," Econometrica, 69, 935-957.

Harvey, Charles M. (1986), "Value Functions for Infinite-Period Planning," Management Science, 32, 1123-1139.

Kirby, Kris N. and Nino N. Marakovic (1995) "Modeling Myopic Decisions: Evidence for Hyperbolic Delay-Discounting within Subjects and Amounts," Organizational Behavior and Human Decision Processes, 64, 22-30.

Krusell, Per, and Anthony A. Smith (2003), "Consumption-savings decisions with quasigeometric discounting," Econometrica, 71, 365-375.

Laibson, David (1997), "Golden eggs and hyperbolic discounting," Quarterly Journal of 
Economics, 112, 443-477.

Loewenstein, George, and Drazen Prelec (1992), "Anomalies in intertemporal choice: evidence and an interpretation, Quarterly Journal of Economics, 107, 573-597.

Luttmer, Erzo G. J., and Thomas Mariotti (2003), "Subjective Discounting in an Exchange Economy," Journal of Political Economy, 111, 1-30.

Mas-Colell, Andreu, Michael D. Whinston, and Jerry R. Green (1995), "Microeconomic Theory," Oxford University Press, New York.

Mazur, James E. (1987), "An Adjusting Procedure for Studying Delayed Reinforcement," in J. E. Mazur, M. L. Commons, J. A. Nevin, and H. Rachlin (eds.), Quantitative Analyses of Behavior, Vol. 5: The Effect of Delay and of Intervening Events on Reinforcement Value, Hillsdale, NJ: Erlbaum, 55-73.

O’Donoghue, Ted, and Matthew Rabin (1999), "Doing it now or later," American Economic Review, 89, 103-124.

Phelps, E.S., and Pollak, R.A. (1968), "On Second-Best National Saving and GameEquilibrium Growth," Review of Economic Studies, 35, 185-199.

Prelec, Drazen (2004), "Decreasing Impatience: A Criterion for Non-stationary Time Preference and 'Hyperbolic' Discounting," Scandinavian Journal of Economics, 106, 511532.

Read, Daniel, and N.L. Read (2004), "Time Discounting over the Lifespan," Organizational Behavior and Human Decision Processes, 94, 22-32.

Rodriguez, Monica L. and A. W. Logue (1988), “Adjusting Delay to Reinforcement: Comparing Choice in Pigeons and Humans," Journal of Experimental Psychology: Animal Behavior Processes, 14, 105-117. 
Samuelson, Paul A. (1937), "A note on measurement of utility," The Review of Economic Studies, 4, 155-161.

Thaler, Richard H. (1981), "Some Empirical Evidence of Dynamic Inconsistency," Economics Letters, 8, 201-207.

Thaler, Richard H., and Shlomo Benartzi (2004), "Save More tomorrow: Using Behavioral Economics to Increase Employee Saving," Journal of Political Economy, 112, 164-187.

Wakker, Peter P., and Daniel Deneffe (1996), "Eliciting von Neumann-Morgenstern Utilities when Probabilities Are Distorted or Unknown," Management Science, 42, 11311150. 